\title{
Revamping the Cytological Quantitation of Lipid Laden Macrophages in Bronchoalveolar Lavage
}

\author{
F Zahra Aly*, MD, PhD \\ Clinical Associate Professor, Department of Pathology, Immunology \& Laboratory Medicine, University of Florida College of Medicine, \\ Gainesville FL32610-0275
}

Corresponding author: F Zahra Aly, Clinical Associate Professor, Department of Pathology, Immunology \& Laboratory Medicine, University of Florida College of Medicine, Gainesville FL32610-0275

\begin{tabular}{|c|c|}
\hline ARTICLE INFO & ABSTRACT \\
\hline Received: 蔧 October 08, 2020 & $\begin{array}{l}\text { Citation: F Zahra Aly. Revamping the Cytological Quantitation of Lipid Laden Macrophages } \\
\text { inBronchoalveolar Lavage. Biomed J Sci \& Tech Res 31(2)-2020. BJSTR. MS.ID.005063. }\end{array}$ \\
\hline
\end{tabular}

\section{Opinion}

Lipid accumulation in alveolar macrophages appears to occur primarily through the phagocytosis of endogenous or exogenous lipids and lipoproteins. Endogenous lipid accumulation within alveolar macrophages is usually associated with bronchial obstruction, with the lipid material derived from lysed cell membranes of cells distal to the obstructive lesion (cholesterol pneumonia).Exogenoussourcesoflipidwithinalveolarmacrophages result from aspiration of gastric contents, gastroesophageal reflux disease or inhalation of lipids through vaping.Other factors such as obesity and elevated serum cholesterol may also influence lipid laden macrophage formation and prevalence, but their role is not yet established.

In cytology, the lipid content in macrophages is determined by applying a lipid stain, Oil Red 0 (1-[2,5-dimethyl-4-(2-5dimethylphenyl) phenyldiazenyl] azonapthalen-2-ol)) to a formalin fixed smear of cells obtained from bronchoalveolar lavage (BAL) fluid. Semi quantitative methods for assessing lipid laden macrophages include scoring cytology samples based on a visual assessment as originally proposed by Corwin and Irwin in 1985[1] and slightly modified by Colombo and Hallberg [2,3].Specifically, one hundred macrophages are scored on a 0 to 4 scale for presence of lipid vacuoles. Out of a maximum lipid laden macrophage index (LLMI) of 400, an LLMI of $<40$ would not suggest aspiration, 4090 would indicate an indeterminate result and $>90$ would be suggestive of aspiration[2].A higher cut off of $>165$ was proposed by Furuya et al.[4].
Another simplified method of semi quantification of lipid laden macrophage quantification was developed by Collins et al. [3].using cells obtained from tracheal aspirates of children up to 3 years of age.The Oil Red 0 stained cytology specimen is assigned a grade of absent (grade 0), low positive (grade 1: 1-25 lipid-laden macrophages), moderate positive (grade 2: 26-50 lipidladen macrophages), and high positive (grade 3: > 50 lipid-laden macrophages).

Theoretically, the presence of exogenous lipid scavenged form the distal airways and alveoli would indicate aspiration of fatcontaining food or liquid, either from dysfunctional swallowing or gastro esophageal reflux.However, studies reach conflicting conclusions regarding the accuracy of LLMI in predicting aspiration [5-7].Furthermore, there are striking differences in LLMI results across different studies, such that the control groups of some studies had higher indices than other studies' aspiration groups $[8,9]$.Thus, LLMI, as currently assessed appears to have insufficient sensitivity and specificity to be clinically useful.

The lack of consensus might be due to procedural related factors as well as the quantification method.Progress is being made in this regard with the publication of American Thoracic Society guidelines for improving the quality and reproducibility of flow cytometry samples obtained from the lung [10].Quantification factors influencing the assessment of LLMI in cytology include marked interobserver variance due to the subjective nature of visual 
quantification.Most critically, the proposed indices do not take into account the proportion of lipid-laden macrophages relative to the whole specimen.

There are several ways in which lipid laden macrophages can be better quantified.Marzahl et al. [11].used a deep learning-based method of annotated cytology from whole slide digital images of BAL to achieve high concordance in quantification of hemosiderophages. Another approach would be to use mass spectrometry whereby the lipid content can be precisely characterized, and its source identified as food based (implying gastric contents). The concentration of lipids in the specimen would serve as a measure of severity of the aspiration.

Flow cytometry of BAL in inflammatory lung disorders has proven to be clinically useful precisely because of the objective quantification of immune cell subsets within the specimen.We have used flow cytometry to assess lipid-laden macrophages by staining alveolar macrophages with fluorochrome labeled CD68.0ur preliminary study utilized wide side scatter to demonstrate lipidladen macrophages that correspond to Grade 3 index by Collins et al.[3]. Flow cytometry would eliminate the influence of confounding factors of pigment (hemosiderin, anthrocotic) laden macrophages, adequacy of the Oil Red 0 stain and obscuring inflammation.

A critical need exists for a more objective, standardized method to quantify lipid-laden alveolar macrophages before its value can be determined in the differential diagnosis of disorders such as asthma, oropharyngeal reflux and vaping.The dramatic increase in vaping and the putative impact it has on lipid laden macrophages has further underscored this need.Of the various options, flow cytometry appears to hold the most promise of delivering such a method but further research needs to be done to validate its utility.

\section{References}

1. Corwin RW, Irwin RS (1985) The lipid-laden alveolar macrophage as a marker of aspiration in parenchymal lung disease. Am Rev Respir Dis 132(3): 576-581.

2. Colombo JL, Hallberg TK (1987) Recurrent aspiration in children: lipidladen alveolar macrophage quantitation. Pediatr Pulmonol 3(2): 86-89.

3. Collins KA, Geisinger KR, Wagner PH, Blackburn KS, Washburn LK (1995) The cytologic evaluation of lipid-laden alveolar macrophages as an indicator of aspiration pneumonia in young children. Arch Pathol Lab Med 119(3): 229-231.

4. Furuya ME, Moreno Córdova V, Ramírez Figueroa JL, Vargas MH, RamónGarcía G, et al. (2007) Cutoff value of lipid-laden alveolar macrophages for diagnosing aspiration in infants and children. Pediatr Pulmonol 42(5): 452-457.

5. Chang AB, Cox NC, Purcell J, Marchant JM, Lewindon PJ, et al. (2005) Airway cellularity, lipid laden macrophages and microbiology of gastric juice and airways in children with reflux oesophagitis. Respir Res 6: 72.

6. Rosen R, Fritz J, Nurko A, Simon D, Nurko S (2008) Lipid-laden macrophage index is not an indicator of gastroesophageal reflux-related respiratory disease in children. Pediatrics 121(4): e879-e884.

7. Nussbaum E, Maggi JC, Mathis R, Galant SP (1987) Association of lipidladen alveolar macrophages and gastroesophageal reflux in children. J Pediatr 110(2): 190-194.

8. Reid Nicholson M, Kulkarni R, Adeagbo B, Looney S, Crosby J (2010) Interobserver and intraobserver variability in the calculation of the lipid-laden macrophage index: implications for its use in the evaluation of aspiration in children. Diagn Cytopathol 38(12): 861-865.

9. Gibeon D, Zhu J, Sogbesan A, Banya W, Rossios C, et al. (2014) Lipid-laden bronchoalveolar macrophages in asthma and chronic cough. Respir Med 108(1): 71-77.

10. Tighe RM, Redente EF, Yu YR, Herold S, Sperling AI, et al. (2019) Improving the Quality and Reproducibility of Flow Cytometry in the Lung. An Official American Thoracic Society Workshop Report. Am J Respir Cell Mol Biol 61(2): 150-161.

11. Marzahl C, Aubreville M, Bertram CA, Stayt J, Jasensky AK, et al. (2020) Deep Learning-Based Quantification of Pulmonary Hemosiderophages in Cytology Slides. Sci Rep 10(1): 9795.
ISSN: 2574-1241

DOI: 10.26717/BJSTR.2020.31.005063

F Zahra Aly. Biomed J Sci \& Tech Res

(C) (P) This work is licensed under Creative

Submission Link: https://biomedres.us/submit-manuscript.php

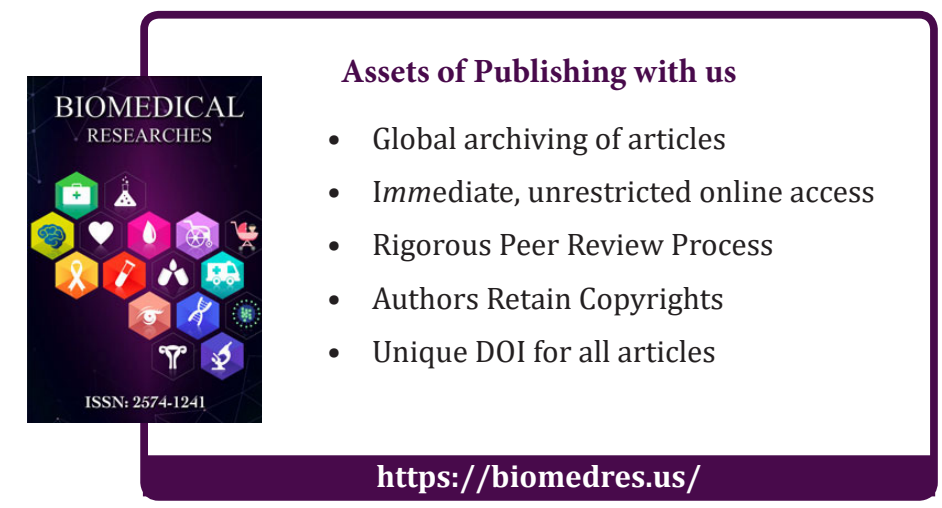

